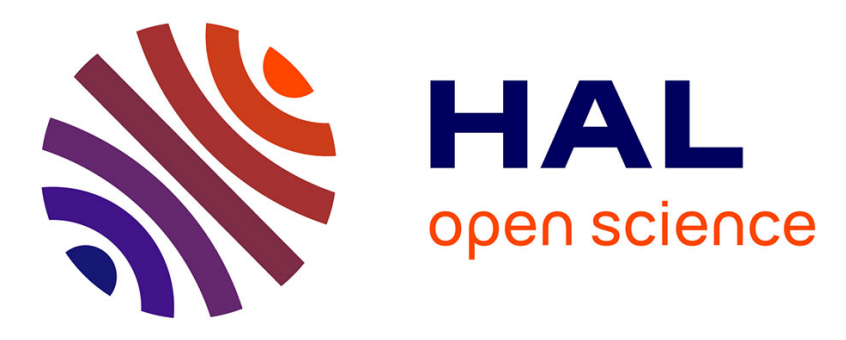

\title{
Current-induced domain wall motion in Ni80Fe20 nanowires with low depinning fields
}

Gregory Malinowski, Andreas Lörincz, Stephen Krzyk, Philipp Möhrke, Daniel Bedau, Olivier Boulle, Jan Rhensius, Laura J Heyderman, Young Jin Cho, Sunae Seo, et al.

\section{To cite this version:}

Gregory Malinowski, Andreas Lörincz, Stephen Krzyk, Philipp Möhrke, Daniel Bedau, et al.. Currentinduced domain wall motion in Ni80Fe20 nanowires with low depinning fields. Journal of Physics D: Applied Physics, 2010, 43 (4), pp.45003. 10.1088/0022-3727/43/4/045003 . hal-00569728

\section{HAL Id: hal-00569728 https://hal.science/hal-00569728}

Submitted on 25 Feb 2011

HAL is a multi-disciplinary open access archive for the deposit and dissemination of scientific research documents, whether they are published or not. The documents may come from teaching and research institutions in France or abroad, or from public or private research centers.
L'archive ouverte pluridisciplinaire HAL, est destinée au dépôt et à la diffusion de documents scientifiques de niveau recherche, publiés ou non, émanant des établissements d'enseignement et de recherche français ou étrangers, des laboratoires publics ou privés. 


\title{
Current induced domain wall motion in $\mathbf{N i}_{80} \mathbf{F e}_{20}$ nanowires with low depinning fields
}

\author{
Grégory Malinowski ${ }^{1}$, Andreas Lörincz ${ }^{1}$, Stephen Krzyk ${ }^{1}$, \\ Philipp Möhrke ${ }^{1}$, Daniel Bedau ${ }^{1,3}$, Olivier Boulle ${ }^{1}$, Jan \\ Rhensius $^{1,2}$, Laura J. Heyderman ${ }^{2}$, Young Jin Cho ${ }^{3}$, Sunae Seo $^{3}$ \\ and Mathias Kläui ${ }^{1}$ \\ ${ }^{1}$ Fachbereich Physik, Universität Konstanz, Universitätsstrasse 10, D-78457, \\ Germany. \\ ${ }^{2}$ Laboratory for Micro- and Nanotechnology, Paul Scherrer Institut, CH-5232 \\ Villigen PSI, Switzerland. \\ 3 Samsung Electronics, San 14-1 Nongseo-dong, Giheung-gu, Yongin-si, Gyeonggi-do, \\ Korea. \\ E-mail: gregory.malinowski@uni-konstanz.de
}

\begin{abstract}
.
In this paper, we report on domain wall motion induced by current pulses at variable temperature in $900 \mathrm{~nm}$ wide and $25 \mathrm{~nm}$ thick $\mathrm{Ni}_{80} \mathrm{Fe}_{20}$ wires with low pinning fields. By using Ar ion milling to pattern our wires rather than the conventional lift-off technique, a depinning field as low as $\sim 2-3$ Oe at room temperature is obtained. Comparison with previous results acquired on similar wires with much higher pinning shows that the critical current density scales with the depinning field, leading to a critical current density of $\sim 2.5 \times 10^{11} \mathrm{~A} / \mathrm{m}^{2}$ at $250 \mathrm{~K}$. Moreover, when a current pulse with a current density larger than the critical current density is injected, the domain wall is not necessarily depinned but it can undergo a modification of its spin structure which hinders current-induced domain wall motion. Hence, reliable propagation of the domain wall requires an accurate adjustment of the pulsed current density.
\end{abstract}




\section{Introduction}

The interaction between a spin polarized current and a magnetic domain wall (DW) in a magnetic nanostructure has attracted much attention during the last decade because of fundamental interest in the underlying mechanisms and also because of promising applications based on DWs such as magnetic memory and logic devices. These applications exploit the recently discovered spin transfer torque mechanism $[1,2]$, i.e. a transfer of spin angular momentum between the conduction electrons and the magnetic moments inside the DW, to manipulate and displace a DW. While the control of the DW dynamics including the depinning mechanism and the motion is of key importance for the reliability of the switching of future devices, it is also of fundamental interest. The depinning mechanism can reveal details of the interaction between the spin-polarized current and the magnetization, as it was predicted to be often accompanied by a spin structure transformation [3]. Furthermore, other key parameters such as the critical current density $[4,5,6,7]$, the DW velocity $[8,9,10]$, and transformation of the DW's structure $[9,10]$ contain information on the relation between the non-adiabaticity and the damping [11].

In order to depin and propagate a magnetic DW, a significant current density of the order of $\sim 1 \times 10^{12} \mathrm{~A} / \mathrm{m}^{2}$ is usually required in the case of $\mathrm{Ni}_{80} \mathrm{Fe}_{20}$ nanowires. This high current density is a drawback for possible applications since it can lead to large temperature rises during current pulse injections due to Joule heating, inducing creation or annihilation of DWs $[12,13]$ or transformations of the DW type $[13,3]$.

Based on theories of spin transfer, assuming that the electron spin follows adiabatically the local magnetization $[14,15,16]$, an intrinsic critical current density independent of the pinning strength is expected. However, large discrepancies between experiments and theory have led theoreticians to introduce a non-adiabatic contribution which acts as a magnetic field on the DW $[17,18]$. Due to this non-adiabatic contribution to the spin transfer torque, the critical current depends on extrinsic properties of the wire and is directly correlated to the pinning strength. As the dynamic propagation field is usually lower than the depinning field [19], the depinning field essentially determines the critical current density. Therefore, a possible approach to reduce the critical current for DW depinning and subsequent propagation would be to reduce the critical field needed to depin a magnetic DW.

In this paper, we investigate the effects of using Ar ion milling to pattern our samples instead of the conventional lift-off approach. This results in very low edge roughness and consequently a low depinning field. Current induced DW motion experiments are carried out at variable temperature. By comparing our results with previous experiments on similar wires with much higher pinning fields, we show that, for our samples, a large reduction of the critical current density is observed which could be attributed to the very low pinning field. Moreover, we find a non-monotonous variation of the critical current density when the temperature is increased due to the competition between thermal activation and the intrinsic temperature dependence of 
the spin torque. Injecting a current pulse with a density larger than the critical density does not necessarily lead to depinning of the DW as DW spin structure transformations occur leading to a stronger pinning of the wall. Therefore tailored pulses are needed for reliable displacement.

\section{Experimental details}

The samples used in this study consist of zigzag shaped nanowires made of $\mathrm{Si} / \mathrm{SiO}_{2} / \mathrm{Py}$ $(25 \mathrm{~nm}) / \mathrm{Au}(2 \mathrm{~nm}) / \mathrm{Cr}(20 \mathrm{~nm})$ with a width of $900 \mathrm{~nm}$, fabricated with Ar ion etching through a mask of Hydrogen silsesquioxane (HSQ) negative resist exposed by electron beam lithography. The HSQ was completely removed during the ion etching as well as approximately half of the Cr layer. Au contacts were added in a second lithography step for magnetoresistance measurements. A scanning electron microscopy (SEM) image of the sample is shown in Fig. 1(a). Before each measurement, the sample is initialized by applying a large magnetic field along the y axis (see Fig. 1(a)). After reducing the field to zero, DWs are generated at the bends of the wires. For this particular geometry, the DW is expected to be of the vortex wall type [20] with a tail-to-tail or head-to-head configuration depending on the initial direction of the magnetic field.

In order to study the influence of a spin-polarized current on the depinning field, we inject current pulses with a pulse length of $50 \mu s$ and increasing amplitude, at distinct values of the magnetic field applied along the $\mathrm{x}$ axis, starting from zero and increasing in steps of 1 Oe or less. The DW is reinitialized with a field after each pulse injection. The depinning field is measured by detecting the increase in the resistance in the area between two contacts close to the bend when the DW is expelled out of the area between these contacts. This resistance change is due to the anisotropic magnetoresistance contribution of the spins in the domain wall, which lowers the resistance when a domain wall is present [21]. The magnetoresistance measurements were carried out in a bath cryostat for temperatures ranging from $4.4 \mathrm{~K}$ up to $240 \mathrm{~K}$.

\section{Results and discussions}

The influence of the injected current on the depinning field is shown in Fig. 1(b). At zero current and a temperature of $4.4 \mathrm{~K}$, a field of 13 Oe is required to move the DW. The zero current propagation field decreases with increasing temperature, showing that the field induced depinning of a DW is a thermally activated process (see also Fig. 2). The depinning field is more than a factor of 3 smaller than the one measured in similar samples but fabricated with a lift-off technique [22]. This reflects the low pinning potential induced by the small edge roughness due to the Ar ion milling fabrication process. When a positive current pulse is injected, the propagation field remains constant below a current density of $\sim 1 \times 10^{11} \mathrm{~A} / \mathrm{m}^{2}$ for every temperature. Only for a cryostat temperature of $4.4 \mathrm{~K}$, a sudden drop of the depinning field is observed for very low current density probably due to some Joule heating as previously observed 
(a)

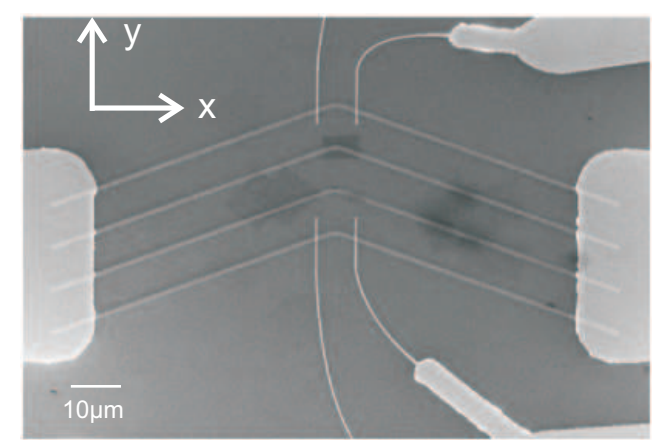

(b)

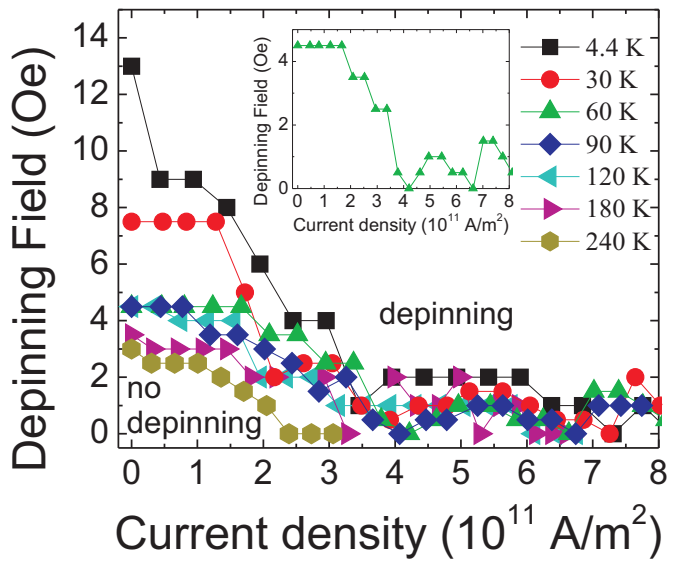

Figure 1. (a) SEM image of the zig-zag shaped wires with a width of $900 \mathrm{~nm}$. (b) Domain wall depinning field as function of the current density for current and field acting to move the wall in the same direction for different cryostat temperatures indicated in the legend. The current pulse length is $50 \mu \mathrm{s}$. For combinations of current and field strength above the lines, the wall is depinned. The inset shows the depinning field as function of the current density for a cryostat temperature of $60 \mathrm{~K}$. Lines are guides to the eye. The standard error on the measured depinning fields is lower than 1 Oe.

[23]. When the current density is increased above $\sim 1 \times 10^{11} \mathrm{~A} / \mathrm{m}^{2}$, an approximately linear decrease of the depinning field is observed until the critical current density $j_{c}^{H=0}$ is reached at which the current moves the domain wall without any applied field (for instance for $60 \mathrm{~K}: 4.2 \times 10^{11} \mathrm{~A} / \mathrm{m}^{2}$, see inset in Fig. 1(b)).

Surprisingly, below $\sim 100 \mathrm{~K}$, the critical current density seems to be independent of the temperature with a value of about $4.0 \times 10^{11} \mathrm{~A} / \mathrm{m}^{2}$. Moreover for temperatures below $240 \mathrm{~K}$, we find for current pulses with a density larger than the critical current density that the DW cannot be moved outside of the area between the contacts when no field is applied (for instance in the inset of Fig. 1(b) for a current density larger than $\left.4.2 \times 10^{11} \mathrm{~A} / \mathrm{m}^{2}\right)$. However, it seems that a reliable depinning is again achieved for a higher current density of about $\sim 7.0 \times 10^{11} \mathrm{~A} / \mathrm{m}^{2}$ which slightly depends on the temperature and possible reasons for this behaviour are discussed later. Moreover, it should be noted that the DW might also propagate without any field applied when a current density of $\sim 9-10 \times 10^{11} \mathrm{~A} / \mathrm{m}^{2}$ is injected.

From these measurements, we extract the critical current density needed to propagate the DW without any applied field as a function of the real sample temperature. This is achieved by appropriately varying the cryostat temperature to compensate the Joule heating as explained previously [23]. The results are plotted in Fig. 2. The critical current density is a factor of five smaller than in similar samples with much higher pinning field, varying from $\sim 4 \times 10^{11} \mathrm{~A} / \mathrm{m}^{2}$ at low temperature down to $\sim 2.5 \times 10^{11}$ $\mathrm{A} / \mathrm{m}^{2}$ at $250 \mathrm{~K}$, which is likely to be a result of the very low pinning potential. To 


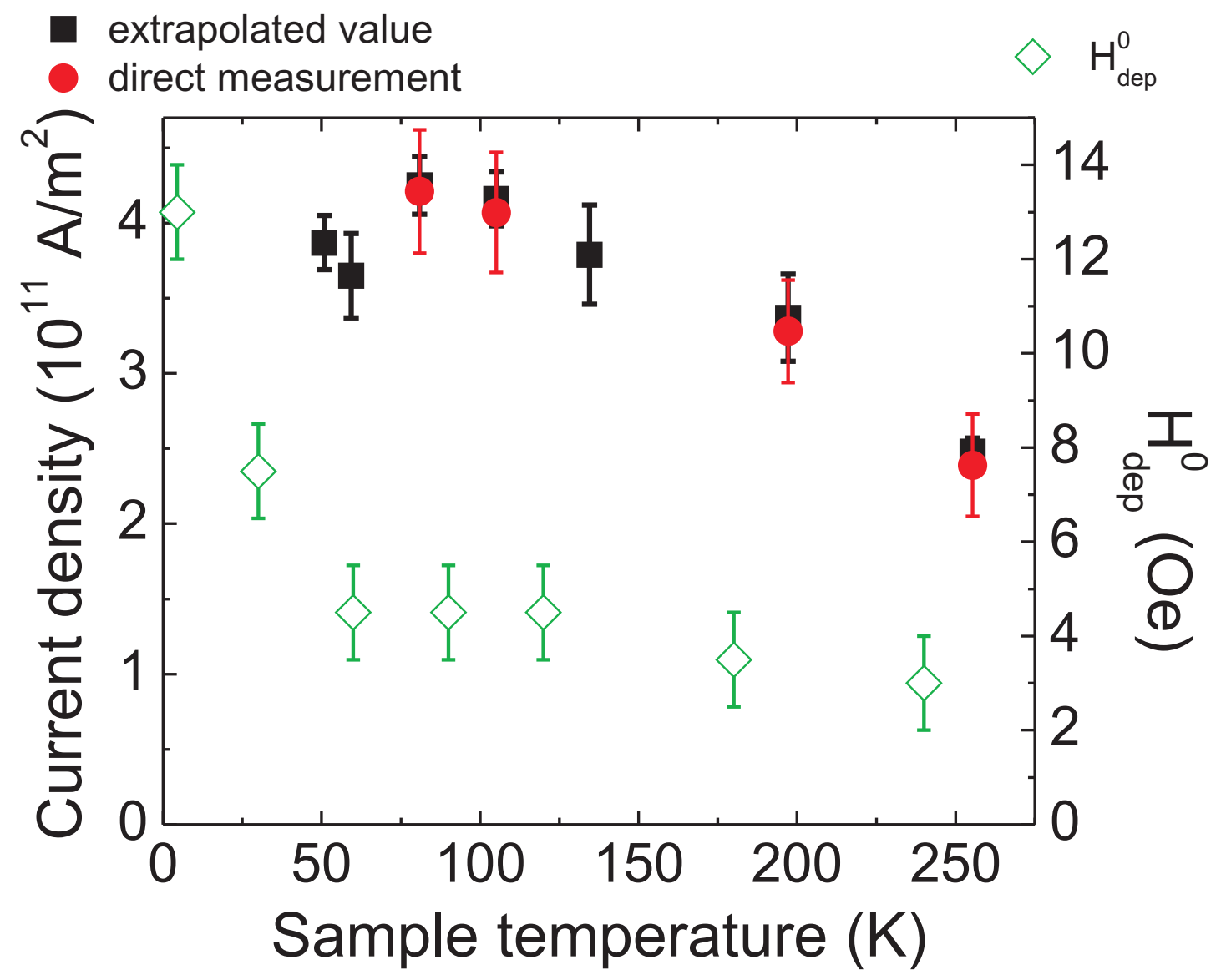

Figure 2. Variation of the extracted critical current density for zero applied field, directly measured (red circles) or extrapolated from a linear fit to the measurements shown in Fig. 1(b) (black squares), and of the depinning field in the absence of injected current, $H_{d e p}^{0}$, as a function of the sample temperature (green open diamonds). Note that the heating leads to a minimum sample temperature of $50 \mathrm{~K}$ for a $4.4 \mathrm{~K}$ cryostat temperature.

corroborate this assumption, we compare these critical current densities and depinning fields with the ones measured on a $\mathrm{Ni}_{80} \mathrm{Fe}_{20}$ wire with a width of $1500 \mathrm{~nm}$ and a similar thickness made by standard lift-off technique. In this wire, in which a lower depinning field would be expected due to its larger width [24], a depinning field of $\sim 10$ Oe is observed [22] at room temperature, so $\sim 4-5$ times higher that observed in the sample made by ion milling. The associated critical current density found is $\sim 1.3 \times 10^{12}$ $\mathrm{A} / \mathrm{m}^{2}$ which is also $\sim 5$ times larger than for the ion milled sample [25]. These results show that the critical current density scales with the pinning strength, indicating that extrinsic pinning governs the depinning field. Moreover, this low critical current density is in very good agreement with recent work reported by Parkin et al. [26]. In fact, they showed that the critical current density for moving a vortex DW in a $\mathrm{Ni}_{80} \mathrm{Fe}_{20}$ nanowire linearly decreases with the pinning field below 15 Oe down to 5 Oe. With these results, we show that the linear decrease persists even for lower pinning fields. 
This bodes well for generating future devices with ultra-low critical current densities by optimizing the wire fabrication process to further reduce the pinning at extrinsic defects. The temperature variation of the critical current density is non-monotonous as previously seen and discussed in Refs. [27, 23]. This is due to the interplay between thermal activation and the intrinsic dependence of the spin torque efficiency on the temperature.

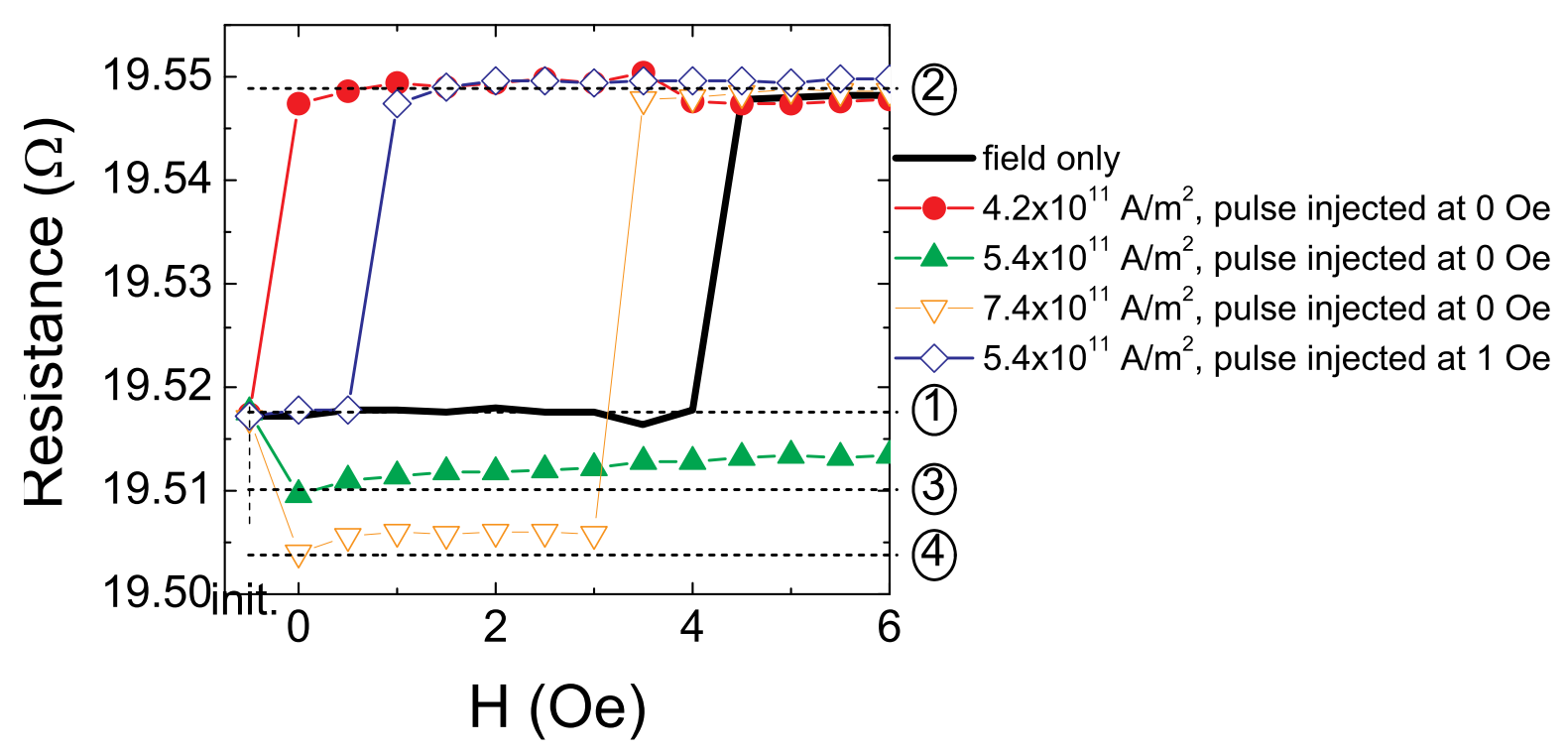

Figure 3. Resistance as a function of the applied magnetic field measured at a cryostat temperature of $60 \mathrm{~K}$. The full black line corresponds to a field induced domain wall depinning measurement without any injected pulse. The red circles correspond to measurement for a single current pulse injected at zero applied field with a current density of $4.2 \times 10^{11} \mathrm{~A} / \mathrm{m}^{2}$. The green up triangles and the orange down triangles correspond to measurement for a single current pulse injected at zero applied field with a current density of $5.4 \times 10^{11} \mathrm{~A} / \mathrm{m}^{2}$ and $7.4 \times 10^{11} \mathrm{~A} / \mathrm{m}^{2}$ respectively. The open blue diamonds correspond to a measurement obtained when a single pulse with the same current current density of $5.4 \times 10^{11} \mathrm{~A} / \mathrm{m}^{2}$ is injected at an applied field of 1 Oe. The points on the abscissa labeled init. show the initial resistance after the DW creation, showing that the same DW structure is reproducibly obtained. The dashed lines represent the resistance levels corresponding to the initial DW structure after its creation (1), to the absence of DW after it has been depinned (2), and to a transformed DW spin structure (3) and (4).

We now discuss the reasons for the fact that a non-monotonous depinning field as function of current density is observed above the critical current (for instance in the inset of Fig. 1(b) for a current density larger than $4.2 \times 10^{11} \mathrm{~A} / \mathrm{m}^{2}$ ). This means that injection of a current pulse larger than the critical current density (the lowest current density at which the wall depins at zero applied field) does not necessarily also lead to a depinning of the DW. This surprising observation might be explained taking into account the periodic DW transformation that depends on the current density. For a given pulse 
length, above a critical current, the DW can transform, yielding a different wall type that is pinned more strongly. As the current is further increased, two transformations can occur during the pulse, transforming the wall back to the original spin structure that can be depinned $[10,12]$. So for an injected pulse with a current density of about $\sim 7.0 \times 10^{11} \mathrm{~A} / \mathrm{m}^{2}$, the DW has a spin configuration similar to the one for the lowest critical current density of $\sim 4.0 \times 10^{11} \mathrm{~A} / \mathrm{m}^{2}$ so that similar depinning is observed. To directly corroborate this explanation direct imaging would be necessary, which is beyond the scope of this paper, but we obtain signs of the transformations from resistance measurements.

To this end, we have carefully analyzed the magnetoresistance traces and we find that in the absence of an applied magnetic field and after injection of a current pulse with a current density larger than the critical current density reported in Fig. 2, a lower resistance state is observed. For example, when we inject a current pulse with a density of $5.4 \times 10^{11} \mathrm{~A} / \mathrm{m}^{2}$ at 0 Oe (Fig. 3 up green triangles), we end up reproducibly at a resistance of $19.510 \Omega$, lower than the initial $19.518 \Omega$ found after creation of the DW. This is likely to be caused by a modification in the DW spin structure. For our wide and thick sample, we are far away from the phase boundary between transverse and vortex DW structures. Thus vortex walls are the most energetically favorable. A transformation upon current injection from a vortex wall to a transverse wall is not only unlikely due to the geometry where a transverse wall would not be stable, but it can also not explain the reduction of the resistance since it has been shown that a transverse wall would lead to a larger resistance $[3,28]$ and not a lower one as observed here. Upon increasing further the injected current density, depinning at zero field is again obtained for a density of $6.6 \times 10^{11} \mathrm{~A} / \mathrm{m}^{2}$. Injection of a current pulse with a larger density at zero apply field leads to a stronger transformation of the DW spin structure resulting in an even larger decrease of the resistance down to $19.504 \Omega$ (down triangles). In that case, the initial change in the resistance correspond to half of the total AMR signal due to the single vortex wall pointing to a noticeable change in the DW spin structure and perhaps to the nucleation of a second vortex wall.

A plausible explanation is that during the pulse injection, a more complex spin structure probably involving the nucleation and anihilation of multiple vortex walls arises. This was observed in a similar geometry by direct imaging in [10] and [13]. In particular Hempe et al. [13] have shown that in such a wide and thick wire, a single vortex wall is not the most favorable spin structure and that more complicated structures such as double or triple vortex walls are formed. Moreover, the energy barrier between the transformation from a vortex wall to a double vortex wall can be similar to the energy barrier to overcome for moving the vortex wall. The presence of a second vortex core in the wire would increase the amount of magnetization perpendicular to the wire and so, to the direction of the current. This would result in a larger magnetoresistive signal than for a single vortex core, giving a lower resistance level as observed here, and also in agreement with micromagnetic simulations reported by Thomas et al. [28].

To corroborate this explanation, we show a similar measurement obtained when 
a single pulse with the a current density of $5.4 \times 10^{11} \mathrm{~A} / \mathrm{m}^{2}$ is injected not at zero field but at a magnetic field of 1 Oe (open blue diamonds in Fig. 3). Before the pulse injection, the resistance is similar to the other measurements, showing that the same DW structure is reproducibly prepared. Then, the small applied field tilts the potential landscape resulting in a lower energy barrier for DW depinning than for a new vortex wall nucleation which is independent of the applied field. Thus the DW is depinned resulting in an increase of the resistance.

\section{Conclusion}

In conclusion, we have measured the combinations of critical current and critical field necessary to move a vortex wall in $\mathrm{Ni}_{80} \mathrm{Fe}_{20}$ wires. By using $\mathrm{Ar}$ ion milling to pattern the samples, a very low pinning potential is obtained due to the low edge roughness resulting in a depinning field of only $2-3$ Oe at room temperature. A critical current density as low as $\sim 2.5 \times 10^{11} \mathrm{~A} / \mathrm{m}^{2}$ at $240 \mathrm{~K}$ is reported and a comparison with other similar samples with much higher pinning shows that the critical current density scales with the pinning strength, pointing to dominating extrinsic pinning. These results highlight the importance of the nanowire fabrication process and the necessity to have the lowest possible pinning potential to ensure a low critical current density. Furthermore, we have observed that injecting a pulse with a current density higher than the critical current density does not necessary lead to a depinning of the vortex wall but induces a modification of the spin structure which hinders its propagation. Therefore, an accurate control of the current density is of paramount importance for a reliable displacement of the DW.

\section{Acknowledgments}

The authors would like to acknowledge the financial support by the DFG through SFB 767, the Landesstiftung Baden Wrttemberg, the European Research Council via its Starting Independent Researcher Grant (ERC-2007-Stg 208162) scheme and the Samsung Advanced Institute of Technology.

\section{References}

[1] Berger L 1986 Phys. Rev. B 331572

[2] Slonczewski J 1996 J. Magn. Magn. Mater. 159 L1-L7

[3] Hayashi M, Thomas L, Rettner C, Moriya R, Jiang X and Parkin S S P 2006 Phys. Rev. Lett. 97 207205

[4] Grollier J, Boulenc P, Cros V, Hamzic A, Vaures A,Fert A and Faini G 2003 Appl. Phys. Lett. 83 509

[5] Vernier N, Allwood D A, Atkinson D, Cooke M D and Cowburn R P 2004 Europhys. Lett. 65526

[6] Kläui M, Vaz C A F, Bland J A C, Wernsdorfer W, Faini G, Cambril E, Heyderman L J, Nolting F and U. Rüdiger 2005 Phys. Rev. Lett. 94106601

[7] Himeno A, Kasai S and Ono T 2005 Appl. Phys. Lett. 87243108 
[8] Yamaguchi A, Ono T, Nasu S, Miyake K, Mibu K and Shinjo T 2004 Phys. Rev. Lett. 92077205

[9] Kläui M, Jubert P O, Allenspach R, Bischof A, Bland J A C, Faini G, Rüdiger U, Vaz C A F, Vila L and Vouille C 2005 Phys. Rev. Lett. 95026601

[10] Kläui M, Heyne L, Backes D, Rüdiger U, Vaz C A F, Bland J A C, Heyderman L J, Cherifi S, Locatelli A, Mentes T O and Aballe L 2006 Appl. Phys. Lett. 88232507

[11] Heyne L, Kläui M, Backes D, Moore T A, Krzyk S, Rüdiger U, Heyderman L J, Rodriguez A F, Nolting F, Mentes T O, Nino M A, Locatelli A, Kirsch K and Matheis R 2008 Phys. Rev. Lett. 100066603

[12] Junginger F, Kläui M, Backes D, Rüdiger U, Kasama T, Dunin-Borkowski R E, Heyderman L J, Vaz C A F and Bland J A C 2007 Appl. Phys. Lett. 90132506

[13] Hempe E M, Kläui M, Kasama T, Backes D, Junginger F, Krzyk S ,Heyderman L J, DuninBorkowski R E and Rüdiger U 2007 Phys. Status Solidi A 2043922

[14] Tatara G and Kohno H 2004 Phys. Rev. Lett. 92086601

[15] Li Z and Zhang S 2004 Phys. Rev. Lett. 92207203

[16] Thiaville A, Nakatani Y, Miltat J and Vernier N 2004 J. Appl. Phys. 957049

[17] Zhang S and Li S 2004 Phys. Rev. Lett. 93127204

[18] Thiaville A, Nakatani Y, Miltat J and Suzuki S 2005 Europhys. Lett. 69990

[19] Beach G S D, Nistor C, Knutson C, Tsoi M and Erskine L 2005 Nature Mater. 4741

[20] Laufenberg M, Backes D, Buhrer W, Bedau D, Kläui M, Rüdiger U, Vaz C A F, Bland J A C, Heyderman L J, Nolting F, Cherifi S, Locatelli A, Belkhou R, Heun S and Bauer E 2006 Appl. Phys. Lett. 88052507

[21] Kläui M, Vaz C A F, Rothman J, Bland J A C, Wernsdorfer W, Faini G and Cambril E 2003 Phys. Rev. Lett. 90097202

[22] Möhrke P, Moore T A, Kläui M, Boneberg J, Backes D, Krzyk S, Heyderman L J, Leiderer P, Bland J A C and Rüdiger U 2008 J. Phys. D: Appl. Phys. 41164009

[23] Laufenberg M, Buhrer W, Bedau D, Melchy P E, Kläui M, Vila L, Faini G, Vaz C A F, Bland J A C and Rüdiger U 2006 Phys. Rev. Lett. 97046602

[24] Kläui M, Vaz C A F, Heyderman L J, Rüdiger U and Bland J A C 2005 J. Magn. Magn. Mater. 29061

[25] Moore T A, Möhrke P and Kläui M unpublished

[26] Parkin S S P, Hayashi M and Thomas L 2008 Science 320190

[27] Dagras P, Kläui M, Laufenberg M, Bedau D, Vila L, Faini G, Vaz C A F, Bland J A C and Rüdiger U 2007 J. Phys. D: Appl. Phys. 401247

[28] Thomas L and Parkin S S P 2007 Handbook of Magnetism and Magnetic Materials Volume 3 942-982 